\title{
Exploring the Benefits of Fingernail Displays
}

Raphael Wimmer

University of Regensburg

93040 Regensburg, Germany

raphael.wimmer@ur.de

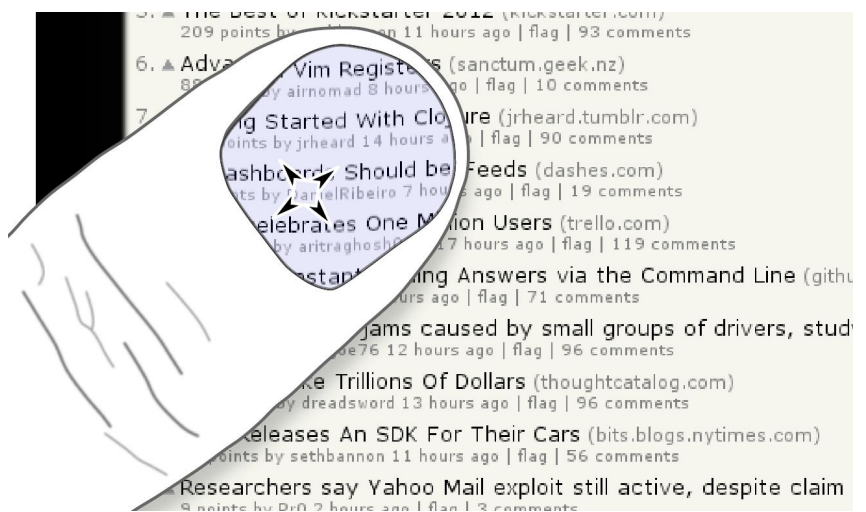

Figure 1: Fingernail displays - artificial fingernails with embedded displays - may help mitigating the fat finger problem or provide always-available, unobtrusive notifications to the user.

Copyright is held by the author/owner(s).

CHI 2013 Extended Abstracts, April 27-May 2, 2013, Paris, France. ACM 978-1-4503-1952-2/13/04.

\begin{abstract}
Fingers are an important interface both to the physical and the digital world. We propose research on artificial fingernails which contain tiny displays and sensors. These fingernail displays greatly supplement other input and output channels, offering novel interaction possibilities. We present three contributions: (1) the general concept and use cases for fingernail displays, (2) a technique for capturing touch events at the fingernails and interaction methods supported by this technique, and (3) an overview of relevant research questions.
\end{abstract}

\section{Author Keywords}

fingernail, displays, touch input, wearable computing

\section{ACM Classification Keywords}

H.5.2 [Information Interfaces and Presentation]: User Interfaces.

\section{Introduction}

In the past few years, touch input has risen to public prominence - due to technological advances and the rise of smartphones with capacitive touchscreens. While research on touch interaction has concentrated on the surface that is being touched, it may be worthwhile to consider information displayed directly on the fingertips. We propose research on fingernail displays - 
fingernail-sized, slightly curved displays that are attached on top of a person's fingernails and are flush with the nail grooves. Fingernail displays offer an unobtrusive, always-available visual output channel on the hand without interfering with the hand's main objective, dexterous manipulation. They can be used as status indicators, magic lenses, or miniature GUls. The size depends on the size of the wearer's fingernails. We did not yet find a reliable source for common fingernail sizes and shapes. However, as a first estimate, displays should be between $10 \mathrm{~mm} \times 10 \mathrm{~mm}$ and $15 \mathrm{~mm} \times 15 \mathrm{~mm}$.

OLED, e-ink and liquid crystal displays are suitable display technologies with significantly different power consumption, update rate, color depth, and flexibility. Adding tiny optical sensors between display and fingernail allows for eyes-free input with one or two fingers.

In the following we review related work, explain how an input channel for fingernail displays can be implemented, discuss possible advantages and limitations of fingernail displays, and present three exemplary use cases. Finally, we document research questions, current state, and future plans for this ongoing research project.

\section{Related Work}

To our knowledge, fingernail displays have not been scientifically investigated so far.

In the the science-fiction movie "Total Recall" $(1990)^{1}$, a receptionist changes the color of her fingernails by touching them with a small wand. In the science fiction movie "The Fifth Element" (1997) ${ }^{2}$, another receptionist does also recolor her fingernails but uses a small box instead, into which she inserts her fingers.

For a design competition sponsored by watch maker Timex in 2004, designers Merana, Schubert, and Takacs submitted concept renderings of a digital watch display to

\footnotetext{
${ }^{1}$ http://www.imdb.com/title/tt0100802

${ }^{2}$ http://www.imdb.com/title/tt0119116
}

be worn on the user's thumbnail [4]. The watch is controlled by touching a metallic area at the rim of the fingernail.

With Skinput, Harrison et al. [2] present an input technique where the user taps the palm of one hand with a finger of the other hand. The resulting sound waves are captured by microphones attached to the user's arm.

From the sound information, the location of the tap is computed. The Skinput prototype employs a GUI that is projected onto the user's palm.

Mascaro et al. [3] found that applying force to the finger pad changes the coloration of the tissue under the fingernail, because blood gets pressed out of the small blood vessels in the finger. They showed that different force levels and force directions result in unique coloration patterns. From these coloration patterns, the direction of forces on the finger pad can be estimated. Mascaro et al. implemented a fingernail-mounted sensor employing small LEDs and photodiodes for measuring the coloration. Their research focused on measuring grasp forces for use in robotics. It inspired our approach to input on fingernails and our hardware implementation.

\section{Input on the Fingernail}

While information displayed on the fingernail offers several advantages, as discussed before, an additional input channel on the fingernail might greatly improve the usefulness of fingernail displays. Such direct input on the fingernail would augment systems which track a finger's position $[1,7]$.

One option for implementing input might be a touch-sensitive layer (e.g., capacitive, resistive) above the fingernail display. This could allow touch interaction and swiping gestures on the display. As the display is very tiny, the available input area is very limited. Additionally, most of the fingernail display would be occluded by the finger touching it. This makes continuous interaction tedious. 


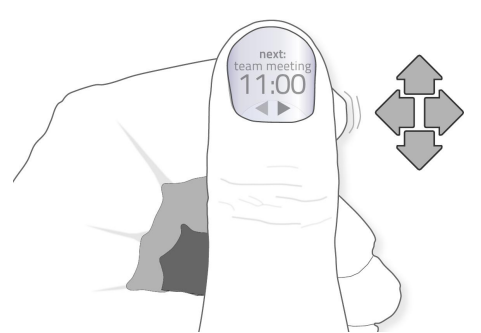

Figure 2: By slightly dragging the pad of the thumb with the index finger in different directions, users can input text or select menu items on the fingernail display.
While it is possible to attach pressure sensors to fingertips ${ }^{3}$, such systems inevitably reduce tactile sensitivity of the finger. We propose an input technique that employs a sensor which is attached to the fingernail but senses pressure on the fingerpad. As demonstrated by Mascaro et al [3], both perpendicular and shear forces applied to the fingerpad can reliably be estimated from changes in the coloration of the tissue under the fingernail. Whereas Mascaro et al. utilized this effect for measuring grasp forces, we plan to detect touch, dragging, and rolling events and use these for explicit and implicit interaction. To this end we are working on an optical sensor which is located between display and fingernail and captures changes in nail bed coloration. We see two potential input modes:

Finger-on-Surface Input Using the approach presented by Mascaro et al, we can detect when an instrumented finger touches a surface with a certain amount of force. This event by itself already allows determining whether the user is doing manual work. We assume that it may also be possible in some cases to find out what the user is doing by comparing the time series of touch events to pre-recoreded training data for known tasks.

Touch-sensitive surfaces allow for further interaction methods: By correlating the timestamps of events from the fingernail with touch events from the surface, it is possible to identify which finger is used for operating a touchscreen, allowing different digital tools to be bound to different fingers. As Mascaro's approach not only detects forces perpendicular to the fingerpad but also shear forces, fingernail sensors can be used to make ordinary touchscreens shear-sensitive. While touching the surface with an instrumented finger, the user can initiate different actions by dragging the finger up/down/left/right. For

\footnotetext{
${ }^{3}$ http://www.pressureprofile.com/
} products-fingertps example, the user might select an object on the touch screen by applying a short downward shear impulse. This impulse would be detected by the fingernail sensor and provided to the touch screen as input event.

Finger-on-Finger Input If two or more fingers are augmented with fingernail sensors, pressing the fingertips together generates simultaneous touch events from all involved fingers. Associating each combination of fingers with a certain action or input symbol allows for unobtrusive, eyes-free mobile input. Measuring shear forces can also be used for turning the thumbpad into a directional input device. Thereby the index finger touches the thumbad and pulls or pushes the thumb's tissue up/down/left/right.

Some of the proposed approaches have previously been implemented using markers on the hands or electrical contacts on the fingerpads. However, fingernail sensors do not require an external tracking infrastructure and do not obstruct tactile senses.

\section{Potential Advantages}

While we are still at the beginning of our research, we see several obvious and potential advantages of fingernail displays that prompted us to investigate further. We plan to verify our assumptions in future research.

Comfort Fingernails and toenails are the only completely rigid body parts not covered by skin. Directly affixing a display to any other body part might make the user feel uncomfortable or irritate the skin. Fingernail displays, in contrast, do not impede tactile senses and do probably not annoy or hinder the wearer if they are flat and flush with the fingernails edges.

Visibility Moving the fingernails into the focus of our field of view is usually very easy and fast. During many manual tasks, - for example when working at a desk - the 
hands are already at least within the peripheral field of view. Usually, both thumbnails are directed towards our eyes while the other fingernails are directed away from us. We assume that in many cases information displayed on the thumbnail is not visible to other people even in close proximity. This makes thumbnails well suited for displaying notifications or context information. The outward-facing fingernails might be used, e.g., for displaying status information to other people, or as optional displays or input buttons.

Effortless Input Putting input and output capabilities at the fingertips allows for quick input and intuitive touch interaction with digital data. As we often look at our hands while doing manual work, putting displays there and offering a rapid input method there - may reduce distraction. When using touch input, our fingers act as pointers for manipulating digital data. A fingernail display can support these interaction, e.g., by making the finger act as a magical lens or a virtual clipboard.

We discuss applications that highlight these potential advantages in the next section.

\section{Challenges and Limitations}

We also see a few unique challenges that need to be taken into account when discussing feasibility and usability of fingernail displays:

Anatomy The size and shape of fingernails varies strongly between persons. Some people have extremely tiny fingernails that would not be suitable for attaching a display. Therefore, we need to find ways to build custom-shaped fingernail displays and accept that there still will be users which can not use these.

As fingernails slowly grow, the attached display will slowly move from its ideal position. When the nail plate is air-tightly covered for a long time, it softens as keratin can not solidify without oxygen contact. This is also a common issue with artificial fingernails. We need to address these issues, e.g., by making the display easily removable and re-attachable.

Social Acceptance It is not yet clear to us whether people would actually wear fingernail displays. There are certainly groups with higher affinity to such attachments than others. On the other hand, fingernail displays might also function as dynamic jewelry, thereby increasing acceptance among less tech-savvy users.

Miniaturization It is not yet clear, whether and how it is possible to manufacture fingernail displays that are thin enough to not impede dexterous manipulation, especially in confined spaces like trousers pockets. At the same time, the displays should be light enough to not cause additional exertion and durable enough that they can be worn during everyday tasks.

Power and Data Transfer The smaller a digital artifact gets, the less power it can store, limiting its wireless use. While we are mainly interested in fingernail displays as a user interface, we feel that we also need to find out how fingernail displays can be powered, and how data can be transmitted between fingernail and other computers.

\section{Usage Scenarios}

Given the interesting properties of fingernail displays, we see several promising applications of which we shortly describe three:

Always-available User Interfaces Having both input and output capabilities directly at the fingertips allows for implementing always-available graphical user interfaces.

Such systems might present notifications about new messages, allow control of a media player, offer little games, and allow people to change their availability status (Figure 2). As the user can glance at and interact with 


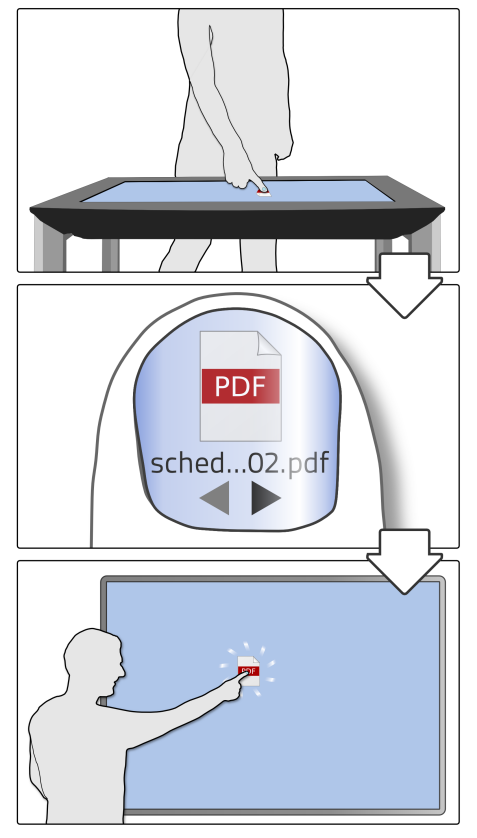

Figure 3: Fingernail displays may be used for implementing user-centered clipboards, allowing for intuitive information transfer between devices. fingernail displays very quickly, we expect them to reduce the amount of time spent with such non-primary tasks.

We are especially interested in the question how fingernail displays compare to - and interact with - wrist-worn displays such as the Sony SmartWatch ${ }^{4}$.

Ubiquitous Clipboard While a growing multitude of smart objects surround us, intuitively transferring data between these objects is still a challenge. Fingernail displays may help here, serving as a ubiquitous clipboard. When the user wants to transfer a file from their (touch-sensitive) computer screen to a wall-mounted display in an adjacent room, they just touch the file on the screen and drag the finger slightly upwards, causing shear forces on the fingerpad. The file gets transferred and is shown as a thumbnail preview on the fingernail display. Then the user walks to the target display and pastes the file by performing a similar gesture on it (Figure 3). Users can copy multiple files to their finger and select the one to be pasted using one of the previously described input methods.

Magic Lenses Fingernail displays can show an augmented image of the area below the finger. This can be used for overlaying visual information onto a touch screen or for showing information about an object being touched or grasped. The information to be displayed could be gathered by additional sensors or wireless interfaces of the objects. For example, touching a battery powered device might show the remaining battery life on the fingernail display, while touching a map might reveal information about the location under the fingertip. We also suspect that a fingernail display that shows the screen contents which are occluded by the finger might mitigate the fat finger effect that reduces input accuracy on touch screens (Figure 1).

\footnotetext{
${ }^{4}$ http://en.wikipedia.org/wiki/Sony_SmartWatch
}

\section{Research Questions}

We suspect fingernail displays might be a useful addition to existing user interfaces. There are a few questions, however, that should be investigated to determine whether fingernail displays have more than just novelty value.

- Which kinds of applications benefit from fingernail displays?

- Which fingers are most suited for augmentation with displays?

- How fast is interaction compared to other Uls such as mobile phones?

- Can fingernail displays mitigate the fat finger problem?

- How can fingernail displays augment other displays, e.g., wrist-worn displays or mobile phones?

- What update rate do displays and sensors need for different applications?

- What is necessary for users to like and wear fingernail displays?

- In which cases are fingernail displays annoying or hindering?

- Which technologies and implementations work best for displays and sensors?

- How important is an exact fit of the displays?

- How can power and data be transferred wirelessly?

To answer these questions, we are implementing prototypes, measuring key properties, and conducting surveys and user studies.

\section{Implementation}

We have built a first prototype of a fingernail display (Figure 4) using an off-the-shelf $96 \times 64$ pixels (121 dpi) OLED display module (Adafruit product ID 326). The display is connected to a Pinguino PIC32MX prototyping board via SPI. The Pinguino is connected to a host 


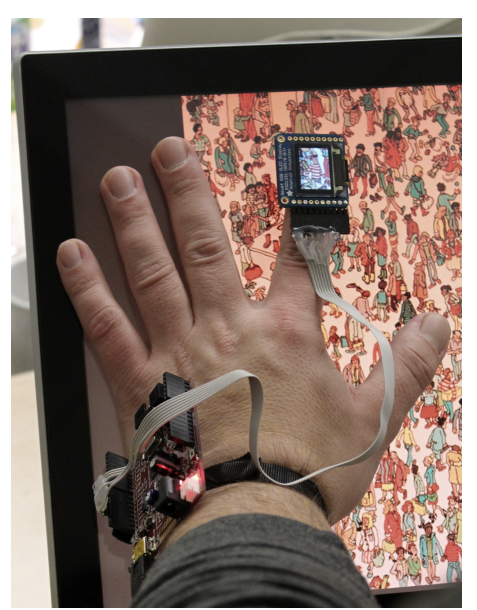

Figure 4: Our first prototype. computer via a serial-over-USB connection. Images are transferred from host computer to OLED display in an uncompressed RGB565 format at an (unoptimized) update rate of $12 \mathrm{fps}$. This prototype does not yet have sensors installed and is quite clunky. However, it helps us in thinking about and discussing applications of fingernail displays. We plan to implement three other prototypes that help us answering our research questions:

a) sensors-only - a prototype without display but five phototransistors. It allows us to refine and test the fingertip input approach without having to bother with display integration.

b) miniaturized - a prototype with sensors and display. We will try to get it as small as a thumbnnail $(15 \mathrm{~mm} \times$ $15 \mathrm{~mm}$ ). It will be powered and controlled via a cable. This prototype will be used for most user studies. c) wireless - a proof-of-concept wireless implementation. With it we plan to demonstrate that fingernail displays can work without cables. We plan to use an e-ink display. Power and data for the display will be transmitted wirelessly.

\section{Future Work}

Using our first prototype, we plan to conduct initial experiments with respect to interaction modalities. In parallel, we will be developing the other prototypes in order to have higher-fidelity hardware for refined studies and test the general feasibility of the more advanced concepts outlined above. Readers may be interested in two other papers on fingernail displays, published simultaneously to this one at CHI'2013 [5, 6]. More information can be found at

https://wiki.mi.ur.de/fingernail.

\section{References}

[1] Harrison, C., and Hudson, S. E. Abracadabra: wireless, high-precision, and unpowered finger input for very small mobile devices. In Proceedings of the 22nd annual ACM symposium on User interface software and technology, UIST '09, ACM (New York, NY, USA, 2009), 121-124.

[2] Harrison, C., Tan, D., and Morris, D. Skinput: appropriating the body as an input surface. In Proceedings of the SIGCHI Conference on Human Factors in Computing Systems, CHI '10, ACM (New York, NY, USA, 2010), 453-462.

[3] Mascaro, S., Chang, K., and Asada, H. Photo-plethysmograph nail sensors: for measuring finger forces without haptic obstruction: modeling and experimentation. In Robotics and Automation, 1999. Proceedings. 1999 IEEE International Conference on, vol. 2, IEEE (1999), 962-967.

[4] Merana, N., Schubert, S., and Takacs, D. TX54 watch concept. http:

//www. core77.com/timex/winners/wearable.asp, 2004.

[5] Su, C.-H., Chan, L., Weng, C.-T., Liang, R.-H., Cheng, K.-Y., and Chen, B.-Y. Naildisplay: Bringing an always-available visual display to fingertips. In Proceedings of the SIGCHI Conference on Human Factors in Computing Systems, CHI '13, ACM (New York, NY, USA, 2013).

[6] Weigel, M., and Steimle, J. Fingernail displays: Handy displays at your fingertips. In CHI '13 Extended Abstracts on Human Factors in Computing Systems, CHI EA '13, ACM (New York, NY, USA, 2013).

[7] Yang, X.-D., Grossman, T., Wigdor, D., and Fitzmaurice, G. Magic finger: always-available input through finger instrumentation. In Proceedings of the 25th annual ACM symposium on User interface software and technology, UIST '12, ACM (New York, NY, USA, 2012), 147-156. 